\title{
Strategy for fast manufacturing of 3D hydrodynamic focusing multilayer microfluidic chips and its application for flow-based synthesis of gold nanoparticles
}

\author{
Yanwei Wang $^{1} \cdot$ Michael Seidel $^{1}$ (D)
}

Received: 1 April 2021 / Accepted: 21 June 2021 / Published online: 5 July 2021

(c) The Author(s) 2021

\begin{abstract}
Fabrication of 3D microfluidic devices is normally quite expensive and tedious. A strategy was established to rapidly and effectively produce multilayer 3D microfluidic chips which are made of two layers of poly(methyl methacrylate) (PMMA) sheets and three layers of double-sided pressure sensitive adhesive (PSA) tapes. The channel structures were cut in each layer by cutting plotter before assembly. The structured channels were covered by a PMMA sheet on top and a PMMA carrier which contained threads to connect with tubing. A large variety of PMMA slides and PSA tapes can easily be designed and cut with the help of a cutting plotter. The microfluidic chip was manually assembled by a simple lamination process. The complete fabrication process from device design concept to working device can be completed in minutes without the need of expensive equipment such as laser, thermal lamination, and cleanroom. This rapid frabrication method was applied for design of a 3D hydrodynamic focusing device for synthesis of gold nanoparticles (AuNPs) as proof-of-concept. The fouling of AuNPs was prevented by means of a sheath flow. Different parameters such as flow rate and concentration of reagents were controlled to achieve AuNPs of various sizes. The sheet-based fabrication method offers a possibility to create complex microfluidic devices in a rapid, cheap and easy way.
\end{abstract}

Keywords 3D hydrodynamic microreactor $\cdot$ Cutting plotter $\cdot$ Laser and heat free $\cdot$ Gold nanoparticles

\section{Introduction}

Microanalytical systems has been developed rapidly in recent decades and offers numerous applications in different scientific and industrial areas. Microfluidics offer unique advantages, including high analytical throughput, enhanced sensitivity, reduced reagent volumes and they are easy to couple with analytical instruments (Cui and Wang 2019). In the early days, microfluidic chips were typically composed of silicon and glass. Although glass and silicon technologies offer high precision, the fabrication methods are complex, time consuming and costly, and cleanroom facilities are

Michael Seidel

Michael.Seidel@mytum.de

Yanwei Wang

Yanwei.wang@tum.de

1 Institute of Hydrochemistry, Chair of Analytical Chemistry and Water Chemistry, Technical University of Munich, Elisabeth-Winterhalter-Weg 6, 81377 Munich, Germany required (Patel et al. 2008). In addition, both materials are fragile and too expensive to be used for disposable devices. Therefore, polymers started to be used as an attractive alternative material, due to their low-cost, wide range of mechanical and chemical properties, flexibility and easy processing possibilities (Song and Lee 2006). The most popular polymers for microfluidic systems are poly(dimethylsiloxane) (PDMS), poly(methylmethacrylate) (PMMA), high-density polyethylene (HDPE), low density polyethylene (LDPE), polyamide 6 and SU-8. One of the most popular and traditional fabrication techniques used for microfluidic chips is soft lithography with PDMS structures made from SU-8 molds (Kim 2008). The basic procedure consists of photolithography, wet-etching and bonding which take a long time from the design to the prototype. Moreover, special training is required for the fabrication of molds and the operation of the equipment. These drawbacks are currently slowing down the development of microchips, especially in research institutions without specialized facilities. Hence, it is crucial to establish rapid prototyping and low-cost alternatives for the fabrication of micro-devices (Faustino et al. 2016). 
Non-lithographic fabrication techniques are a potential alternative to produce micro-devices for researchers who have difficulty to access specialized fabrication facilities and equipment. Rapid cutting by a plotter, also known as Xurography, is one of the non-lithographic techniques using a drag knife printer to generate the master molds, or to directly fabricate the micro-devices (Bartholomeusz 2005). Compared with other non-lithographic methods such as LaserJet (Chen et al. 2016b, 2019), inkjet printers (Vullev et al. 2006) and 3D printing (Chen et al. 2016a), using a cutting plotter for creating channels in foils is significantly cheaper, requires no pumping system, and leaves no combustion residues. The independently pre-cut layers can be bonded together to form channels and microfluidic features (Jafek et al. 2018). Two of the most common and straightforward bonding methods are adhesives and thermal bonding. Thermal bonding is not possible with all materials and the main disadvantage of thermal bonding is the possible warping of features from the heating or cooling. Except for 3D printing, layered microfluidic systems which consist of coated adhesives foils are the fastest and easiest way to fabricate microfluidic channels for most applications. 3D printed microfluidic devices are limited to straight channels and the way to remove the support material could damage the channel (Migneault et al. 2009). Transparent materials can be used for laminated chips to apply chemiluminescence or fluorescence readout systems and it is possible to observe the inside of a microfluidic channel. Recently used microfluidic chips manufactured by the lamination method are summarized in Table 1.

Synthesis of nanoparticles is a popular application for microchips with narrower size distributions and faster reaction rates compared to conventional batch synthesis (Gomez-de Pedro et al. 2010; Shalom et al. 2007; Wagner et al. 2004; Wagner and Kohler 2005). However, significant fouling of the micro-channels is often observed due to deposition of nanoparticles onto the reactor walls (Wagner and Kohler 2005). In an ideal flow focusing device, the central flow should be compressed both horizontally and vertically to obtain uniform fluid velocity which is also called three-dimensional (3D) focusing. In this case, sheath streams can insulate nanoparticles from channel walls and fouling can be avoided (Rhee et al. 2011).

In this work, we propose an easy, cheap, rapid and universal way for the fabrication of laminated PMMA-based 3D flow-focused microreactors. The PMMA sheets and double-sided PSA tape layers were cut using a low-cost cutting plotter. The layers were then assembled with the aid of an alignment tool. 3D microfluidic structure was bonded by a lamination process using mild pressure with a roller and foils coated with an adhesive. Our strategy is to develop highly flexible plastic microreactors without masks and molds (Bemetz et al. 2018). The advantage is that cleanroom requirements due to the absence of lithographic methods are eliminated. Therefore, this technique is adaptive for research institutions without specialized microsystem engineering of lab-on-chip devices. For proof-of-concept, rapid fabrication technique of microfluidic device is applied to produce 3D hydrodynamic focusing microreactors for synthesis of gold nanoparticles (AuNPs) without fouling. The flow-based reaction was based on the online reduction of $\mathrm{Au}$ (III) with ascorbic acid (AA). AuNPs were synthesized at room temperature, unlike with citrate solutions, where heating is required (Shi et al. 2017). Compared with the strong reducing agent sodium borohydride $\left(\mathrm{NaBH}_{4}\right)$, AA cannot react with water, and therefore, no gas is generated. AuNPs were capped with polyvinylpyrrolidone (PVP) for stability. It could be shown by UV-Vis spectroscopy that reproducible AuNPs were synthesized. The concept of flow-based modulation of size and properties for AuNPs was implemented by the 3D hydrodynamic focusing microreactor (Bandulasena et al. 2017; Jun et al. 2012).

Table 1 Summary of laminated method for fabrication of microfluidic chips

\begin{tabular}{|c|c|c|c|c|}
\hline Material & Cutting methods & Bonding methods & Drawbacks & Refrences \\
\hline $\begin{array}{l}\text { Laminating pouches (Scotch } \\
\text { TP3854) }\end{array}$ & Cutting plotter & Thermal lamination machine & $\begin{array}{l}\text { Warping of features from the heat- } \\
\text { ing or cooling }\end{array}$ & $\begin{array}{l}\text { Hernández- } \\
\text { Rodríguez et al. } \\
\text { (2020) }\end{array}$ \\
\hline $\begin{array}{l}\text { Polyethylene terephthalate (PET) } \\
\text { film }\end{array}$ & Cutting plotter & Thermal lamination machine & & Levis et al. (2019) \\
\hline Film of fluoropolymers & Cutting plotter & Heat and pressure & & Hizawa et al. (2018) \\
\hline $\begin{array}{l}\text { Poly(dimethylsiloxane) (PDMS) } \\
\text { membranes }\end{array}$ & Cutting plotter & Plasma bonding & Time consuming & Cosson et al. (2015) \\
\hline Polymethylmethacrylate (PMMA) & $\mathrm{CO}_{2}$ laser & Double-sided tape & Bulges form around microstructure & Chen et al. (2019) \\
\hline $\begin{array}{l}\text { Acrylic sheets and Hybrislip }{ }^{\mathrm{TM}} \\
\text { sheets }\end{array}$ & $\mathrm{CO}_{2}$ laser & Adhesive transfer tapes & $\begin{array}{l}\text { and the laser leaves significant } \\
\text { debris and pyrolysis products }\end{array}$ & Nath et al. (2010) \\
\hline Polymethylmethacrylate (PMMA) & $\mathrm{CO}_{2}$ laser & Thermocompressor & & Chen et al. (2016b) \\
\hline
\end{tabular}




\section{Materials and methods}

\subsection{Materials for the fabrication of microreactor}

The microfluidic reactor was constructed out of three layers of PMMA sheets, three layers of double-sided PSA tapes and one PMMA carrier sheet. The PMMA sheets with a thickness of $0.2 \mathrm{~mm}$ were supplied by modulor material total (Berlin, Germany). The PSA tape (ARcare 90,106) was bought from Adhesive Research (Glen Rock, PA, USA). The carrier sheet with a thickness of $10 \mathrm{~mm}$ was fabricated by our in-house workshop. The fabricated PMMA carrier contained threads $\left(1 / 4^{\prime \prime}-28 \mathrm{UNF}\right)$ to allow the connection with PTFE tubing.

\subsection{Devices and software for fabrication of microchip}

The layout of the microreactor was designed with the software CorelDRAW. The CorelDRAW plug-in Cutting Master 3 from Graphtec Corporation (Yokohama, Japan) was used for communication with the cutting plotter. The digital cutting plotter (Graphtec CE6000-40) was supplied by Graphtec Corporation (Yokohama, Japan) to slit chips into sheets. Cutting conditions and settings were executed via the Cutting Master 3. Programmable resolution was $0.025 \mathrm{~mm}$, and the media type was up to $0.25 \mathrm{~mm}$ thickness. All sheets were assembled in an alignment tool which was supplied by our in-house workshop.

\subsection{Synthesis of AuNPs}

PVP-capped colloidal AuNPs were synthesized by the reduction of tetrachloroaurate (III) ions via AA as described elsewhere (Luty-Błocho et al. 2017). Gold(III) chloride trihydrate $\left(\mathrm{HAuCl}_{4} \cdot 3 \mathrm{H}_{2} \mathrm{O}\right)(\geq 99.9 \%$ trace metal basis $)$ and reagent grade $(\geq 99 \%)$ crystalline L-ascorbic acid (AA or $\mathrm{AsH}_{2}$ ) were supplied from Sigma Aldrich (USA). The gold precursor stream was $1 \mathrm{mM}$ aqueous $\mathrm{HAuCl}_{4}$ solution containing 1\% (w/v) PVP with average MW 29,000 (powder, Sigma Aldrich, Germany) as the capping agent. The reducing agent stream was $20 \mathrm{mM}$ aqueous AA solution with $0.2 \mathrm{M} \mathrm{NaOH}$ solution (Sigma-Aldrich, reagent grade, $\geq 98 \%$, pellets). The $\mathrm{pH}$ of the solution was measured with a FiveEasy pH meter FP20 (Mettler Toledo, Columbus, OH, USA). This reaction was achieved by a 3D flow focusing the AA sheath streams to the gold precursor stream. Before each synthesis process, the microreactor was flushed twice, first with $0.01 \%$ PVP solution to push all bubbles out then the second time with a portion of the reducing reagents to change the $\mathrm{pH}$ in the microreactor channel. The syringes then pushed the reagents into the channel at a controlled flow rate ratio (gold precursor: reducing reagents, 1:10). The synthesized AuNPs were then collected in a vial at the outlet of the microreactor. AuNPs were synthesized using reducing agents with different $\mathrm{pH}$ by adding different amounts of $0.2 \mathrm{M} \mathrm{NaOH}$. The $\mathrm{pH}$ of the original reducing agent was adjusted to 4.30, 5.66, 7.10, 7.61,10.32, 10.91 by adding $200 \mu \mathrm{l}, 400 \mu \mathrm{l}, 510 \mu \mathrm{l}, 520 \mu \mathrm{l}, 600 \mu \mathrm{l}$ and $700 \mu \mathrm{l}$ $0.2 \mathrm{M} \mathrm{NaOH}$ to $5 \mathrm{ml} 20 \mathrm{mM}$ AA solution, respectively. Various flow rates and concentrations of reagents were also investigated.

Reagents were supplied to the microreactor via three glass syringes (Innovative Labor Systeme GmbH, Germany) connected to a six-port valve (Cavro Smart Valve, Tecan Group Ltd., Switzerland). One port of each valve was connected to an inlet of the microreactor, one to the waste to clean the syringes, and the remaining four ports were used for the uptake of reagents and cleaning solution. The glass syringes were operated by three custom made pumps (GWK Präzisionstechnik, Munich, Germany) which were controlled with Matlab (The MathWorks Inc., USA) by a host computer connected via an Ethernet cable. The reaction process was observed and recorded using a microscope camera $(1.3 \mathrm{M}$ pixels, $10 \times, 60 \times, 100 \times$ magnification) (Traveler USB Mikroskop, Supra, Germany). The synthesis and washing processes with different conditions can be controlled and modified in the Matlab program. In this way, all operations could be performed in an automated way. The combined setup is shown in Fig. 1. All waste was collected by a specific company and disposed of in an environmentally friendly way.

\subsection{Characterization of AuNPs}

A SPECORD 250 PLUS UV/Vis spectrometer (Analytik Jena, Jena, Germany) was used to record the UV-Vis

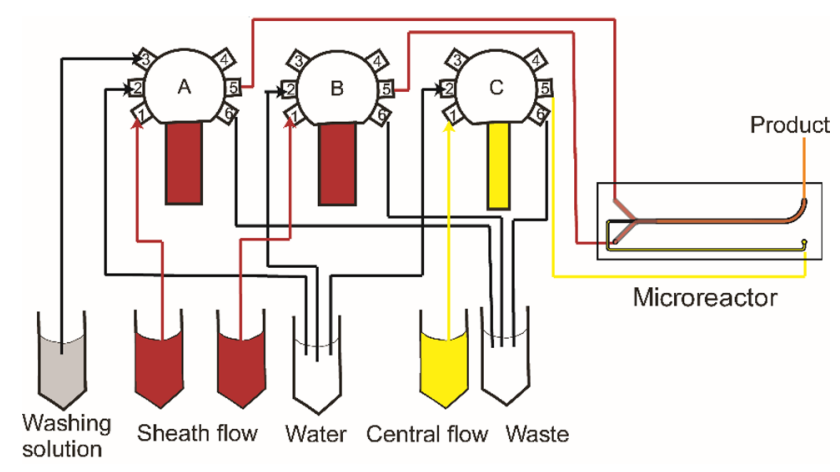

Fig. 1 Scheme of 3D hydrodynamic focusing microreactor connected to reactant streams. Reagents were supplied to the microreactor via three glass syringes connected to a 6-port valve. One port of each valve was connected to an inlet of the microreactor, and other ports were used for the uptake of reagents, cleaning solution. The setup was controlled automatically by Matlab 
absorption spectra of the gold nanoparticle suspensions. The spectrometer uses a deuterium lamp and a halogen lamp to generate electromagnetic waves of ultraviolet and visible wavelengths. A beam of light with a wavelength ranging from 400 to $900 \mathrm{~nm}$ was used for measurement. Disposable polystyrene cuvettes (UV cuvette semi-micro, 1.5-3.0 ml, BRAND GMBH, Germany) were used for containing samples and reference. Since all solutions were prepared in ultrapure water, ultrapure water was used as the reference sample. Scanning electron microscopy (SEM) images were acquired on a model Sigma 300 VP microscope (Zeiss Gemini). The samples were analysed with an InLens detector using an acceleration voltage of $10 \mathrm{kV}$, a $30 \mu \mathrm{m}$ aperture and a working distance of about $1.4 \mathrm{~mm}$.

\section{Results and discussion}

\subsection{Design and fabrication of a microreactor for the online synthesis of AuNPs}

Fouling of nanoparticles by unwanted deposition on surfaces occurs regularly in microstructured devices (Schoenitz et al. 2015). The hydrophobization of inner walls of the reactor through silanization is a general method to suppress fouling in glass chips (Bandulasena et al. 2017; Wagner and Kohler 2005). PMMA is a hydrophobic polymer, easy to handle and process with low cost. Moreover, it can be easily modified into a super-hydrophobic PMMA film (Ma et al. 2007). Hydrophobic material suppresses fouling within the inner walls of the reactor due to an increase in slip boundary conditions which reduce wetting and enhance shear rate on the walls (Rothstein 2010). Besides the material itself, the application of a 3D hydrodynamic flow by focusing the central stream with the sheath streams, as shown in Fig. 2a, is supposed to prevent fouling in microfluidic devices. A separation between the central stream and the channel walls can be achieved, thereby reducing particle-wall interactions. The microfluidic reactor was constructed of seven layers of alternating PMMA sheets and PSA tapes. Figure $2 b$ depicts the scheme of the laminated microfluidic device. The central layer had a channel with a width of $0.7 \mathrm{~mm}$ in the first part for the inlet of central flow and then extended to a width of $1.5 \mathrm{~mm}$ for the mixing. This layer came from a PSA tape with a thickness of $0.12 \mathrm{~mm}$. Then, the tape was laminated between two PMMA sheets. Afterwards, two PSA tapes with a channel for sheath flow were applied on both sides. The device was closed by a $0.5 \mathrm{~mm}$ PMMA sheet on the top and a PMMA carrier with a thickness of $10 \mathrm{~mm}$ on the bottom with three inlet and one outlet drill threads holes (1/4"-28 UNF). The main dimensions are shown in Figure S1. The difficulty in designing and fabricating 3D hydrodynamic focusing microreactor was solved by the simple assembly process of layering pre-cut PMMA sheets and PSA tapes.

One layer of the microfluidic reactor was processed by a cutting plotter, as shown in Fig. 3a. (i) A double-sided PSA tape was adhered onto the repositionable adhesive surface of a reusable cutting mat. (ii) After a customized microfluidic reactor design was generated, the drawing was loaded onto the cutter software. The cutting plotter cut the design out. (iii) While the cut-out double-sided PSA tape was still adhered onto the reusable cutting mat,
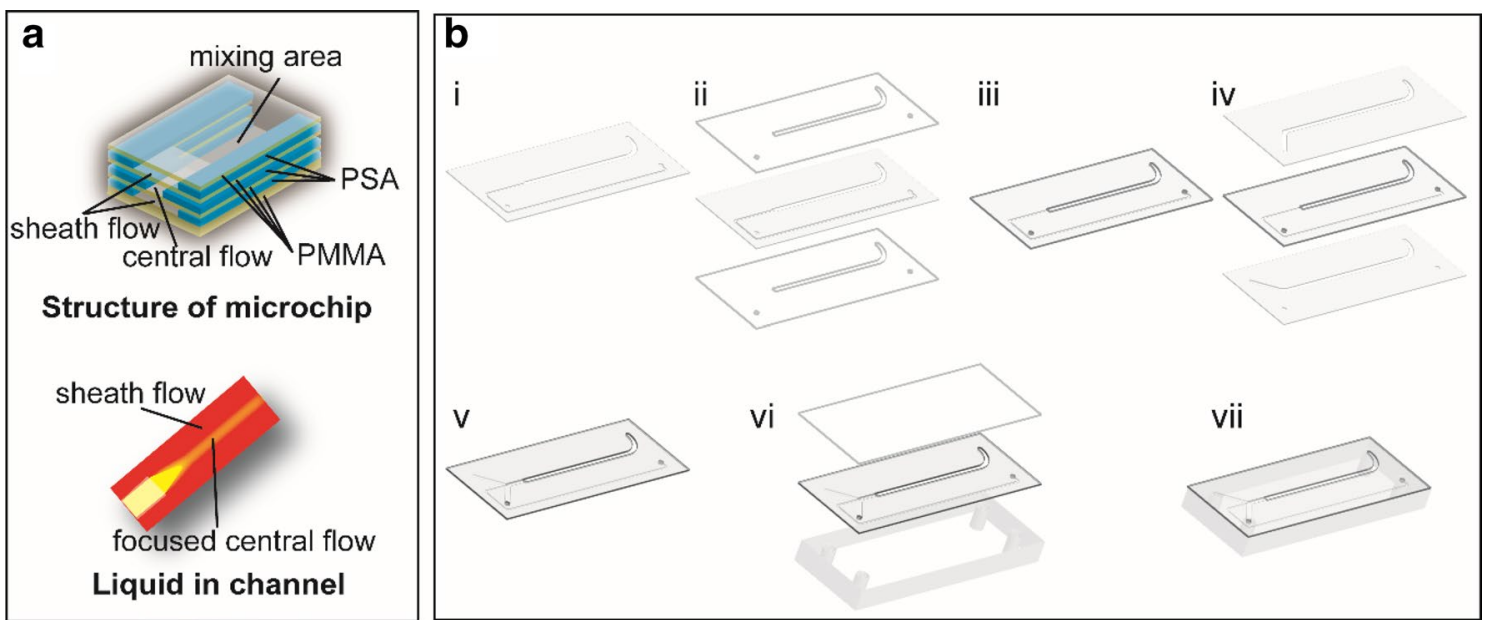

Fig. 2 a Scheme of the 3D microfluidic reactor designed for creation of a central stream by hydrodynamic focusing with a sheath stream. The sheath flow channels were wider, and they were applied on both top and bottom of central flow channel. Therefore, the central flow was focused horizontally and vertically to the centre. b Preparation scheme for the laminated 3D flow focusing device: (i) central layer was cut from PSA tape with digital cutting plotter; (ii-iii) central layer was sandwiched between two PMMA sheets; (iv-v) sheath stream support channels were applied to the top and bottom of the central channel; (vi-vii) cover slide and PMMA carrier with threaded holes for tube connection were applied to yield the final completed device 


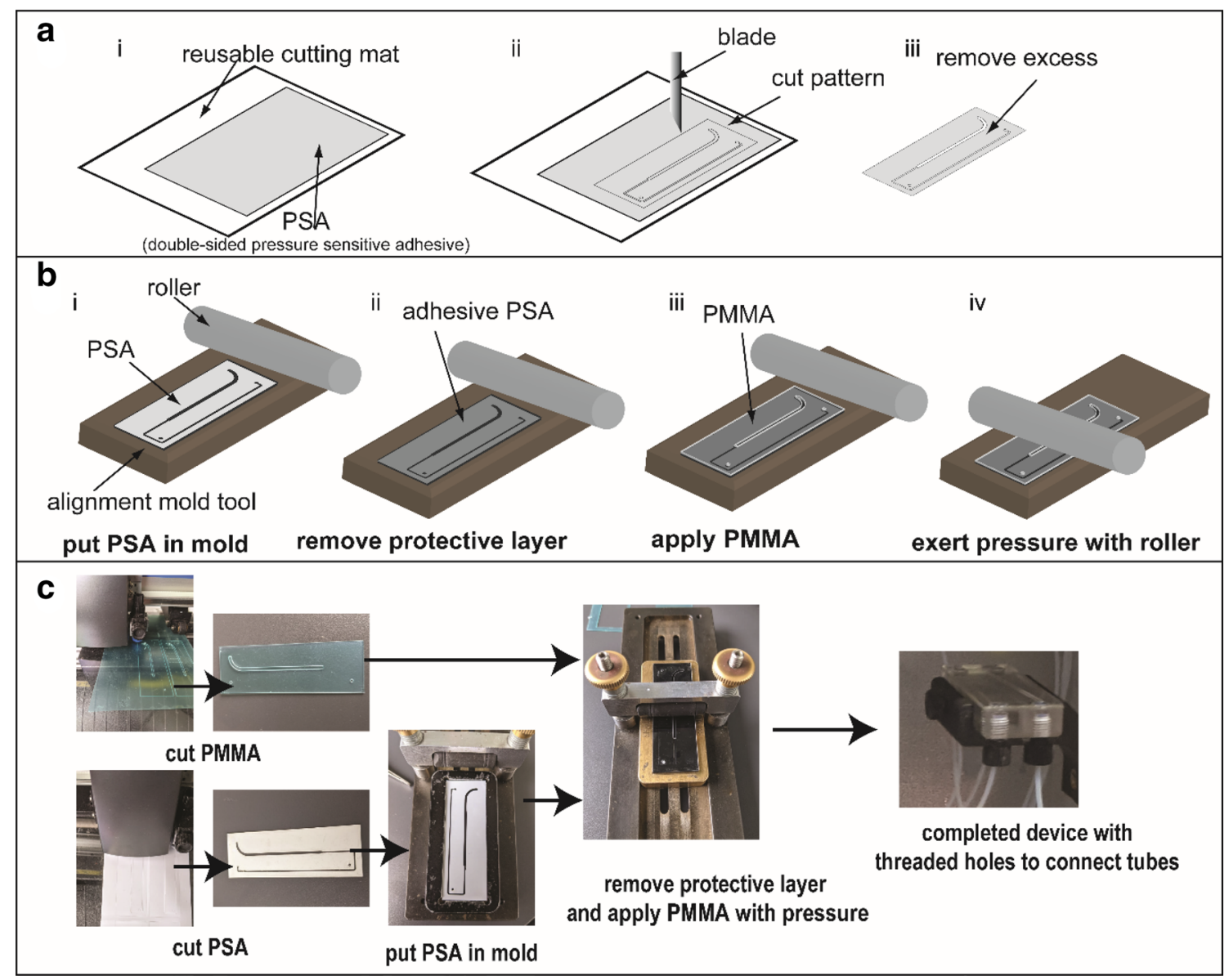

Fig. 3 a Process scheme for cutting one layer of the chip: (i) the PSA attached on a reusable cutting mat, (ii) the pattern was cut out by cutting plotter, (iii) remove excess part and get the final chip layer. b Process scheme of assembling layers together. (i) PSA tape is put in the mold. (ii) The top protective layer of the PSA tape is peeled

the cut pattern was carefully removed using tweezers. The PMMA sheets were created using the same method. The sheets were manually assembled together with a mold tool for alignment. The mold had the same size as all the sheets which ensured that the individual layers were aligned correctly. The roll above can be adjusted to exert pressure on the sheets and made sure that the microchip was sealed. The whole process is shown in Fig. 3b. (i) PSA tape is put in the mold. (ii) The top protective layer of the cut double-sided PSA tape is peeled away revealing the top permanent adhesive surface. (iii) The pre-cut transparent PMMA sheet is applied onto the top of the permanent adhesive surface in the mold. (iv) Pressure is exerted by the roller to bind them together and make sure not to cause any air bubbles or wrinkles in the whole assembly. 3D microfluidic devices can be fabricated by repeating the away revealing the top permanent adhesive surface. (iii) PMMA sheet is applied onto the top of the permanent adhesive surface in the mold. (iv) Pressure is exerted by the roller to bind them together. c Process image for cutting layers and assemble them together to get a complete device

above steps. The images of each step for fabrication of microreactor are shown in Fig. 3c.

\subsection{Comparison of 2D and 3D microreactor}

Our first flow-based microreactor was designed as depicted in Fig. 4a. First half of the central PSA layer was used as inlet for the gold precursor and second half for mixing of all the reagents. Reducing agents were pumped through the PSA channel above and below the central layer. However, when it was used for the online synthesis of AuNPs, there were some challenges to be addressed. First of all, some small bubbles stuck in the interface and then converged into a big one, as shown in Fig. 4a. The big bubble could not be flushed out by liquid. In this case, it interfered with the flow pattern and interrupted the continuous production. Second, the central PSA layer was so thin that it did not offer enough space for mixing. Synthesized AuNPs easily fouled on the wall 
Fig. 4 Design of microreactors with inserted pictures of interfaces and mixing channels. a 2D microfluidic reactor which generated bubbles and fouling. b 3D microfluidic reactor and image of the flow channels showing no fouling. The reactant stream flow rates $\left(\mathrm{HAuCl}_{4} /\right.$ AA) were $1 / 10 \mu \mathrm{l} / \mathrm{s}$. The $\mathrm{pH}$ of the reducing agent was 8
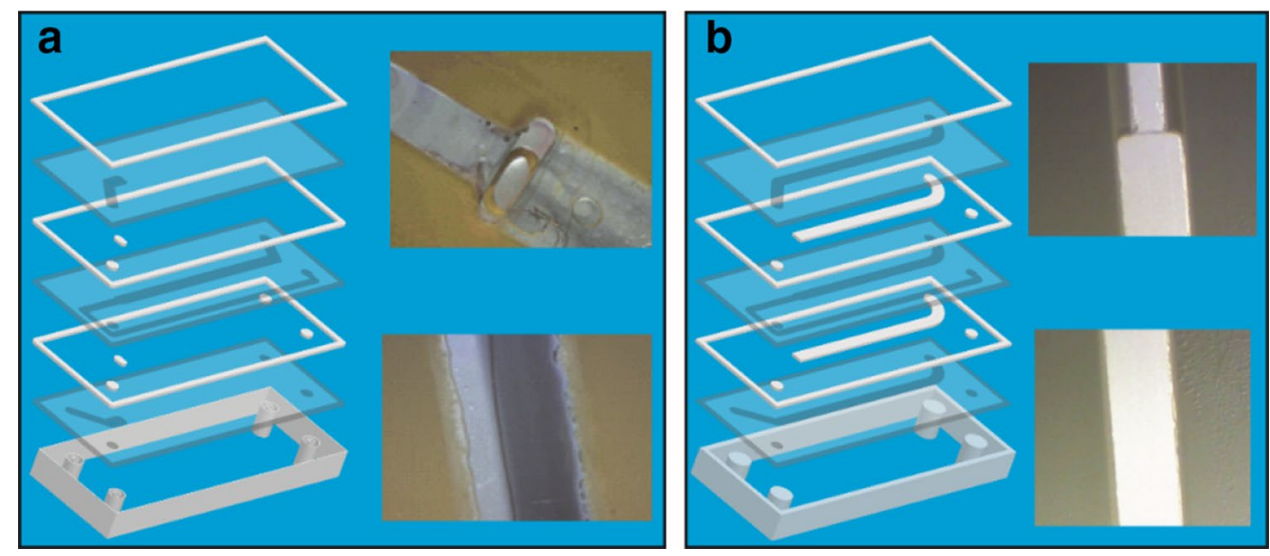

and finally blocked the channel. In addition, all the reagents were directly mixed together in the interface and the sheath stream was not able to focus the central stream. All these issues indicate that this type of microreactor rather has the properties of a 2D chip even though it technically is defined as $3 \mathrm{D}$. The microreactor was then modified by extending the sheath flow channel to the outlet. The PMMA layer between the central flow layer and sheath flow layer was opened from the interface for mixing, as shown in Fig. 4b. In this case, no bubbles blocked the channel. In addition, the mixing area was enlarged and offered more space. Also, the corner was changed to a curve which allowed a stable flow and reduced small bubbles. In the 3D microfluidic reactor, the central stream was focused in the centre both vertically and horizontally. As no AuNPs can contact the channel wall, the microreactor was clean all the time as shown in Fig. $4 \mathrm{~b}$.

\subsection{Effect of various interfaces}

The interface was defined as the region, where the central stream and the sheath streams start to mix (Fig. 5a, b). This is a crucial part, where the reaction begins to take place and is easy to get fouling. Moreover, some bubbles could accidentally come into the system from liquid or syringes and the flow could be interrupted if they were blocked in the interface. Three different interfaces were compared, semicircle (Fig. 5c), bevel (Fig. 5d) and right angle (Fig. 5e). It was shown that there was some fouling on the semi-circular interface (Fig. 5c), because this shape was easy to absorb and converge bubbles. The flow pattern was disturbed there and a portion of reducing agent even flowed back to react with the gold precursor and caused fouling. To reduce dead volume and solve the bubble trapping issue, the bevel interface was designed. However, there was still a problem for the bevel one, a portion of the central gold precursor flowed along the wall in the mixing area and the generated AuNPs have the chance to foul on the wall, as shown in Fig. 5d. Therefore, the interface was changed to right angle (Fig. 5e). Small bubbles were easily flushed away, because the right angle did

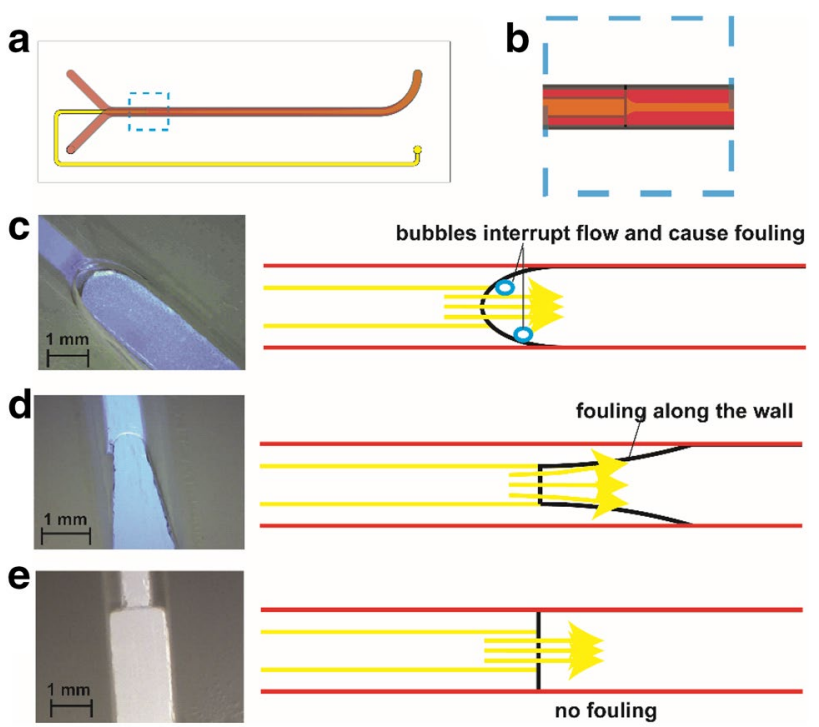

Fig. 5 a Scheme of microreactor with central flow $\left(\mathrm{HAuCl}_{4}\right.$, yellow) and sheath flow (AA, red) mixing in interface, $\mathbf{b}$ scheme of interface and $\mathbf{c}-\mathbf{e}$ images and schemes of different designs of interfaces (yellow line with arrow in schemes represents central stream). The reactant stream flow rates $\left(\mathrm{HAuCl}_{4} / \mathrm{AA}\right)$ were $1 / 10 \mu \mathrm{l} / \mathrm{s}$. The $\mathrm{pH}$ of the reducing agent was 8

not block them. Moreover, the central stream flowed forward and entered the centre of the mixing area without having a chance to touch the mixing wall. How the central stream was focused to the centre with right angle interface is shown in Figure S2. The effect of flow rates on the focus stream was also investigated in Supplementary Materials.

\subsection{Online synthesis of AuNPs}

The optimized 3D microfluidic reactor was used for online synthesis of PVP capped AuNPs. The synthesis reproducibility was characterized using surface plasmon resonance (SPR) band intensity and shift as indicator parameters by UV-Vis spectra. 5 syntheses were carried out using the same device and same reactant conditions. As shown in Fig. 6a, 


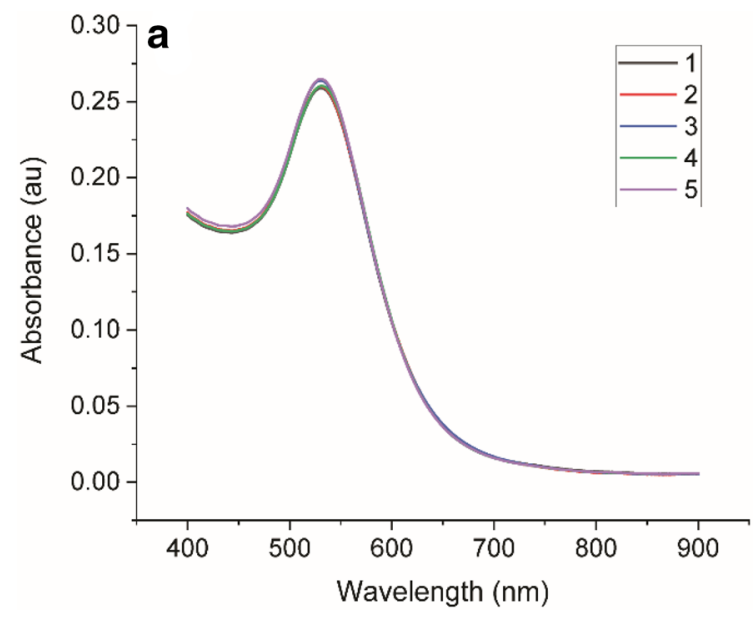

Fig. 6 UV - Vis spectra of the AuNPs synthesized in the a 3D microreactor and the $\mathbf{b} 2 \mathrm{D}$ microreactor. 5 syntheses were carried out using the same device and same reactant conditions and the number in the

the absorbance spectra of these five syntheses are almost overlapping each other with a band located about $531 \mathrm{~nm}$ and absorbance of $0.26 \pm 0.006 \mathrm{au}$. It demonstrated the robustness of the proposed flow system indicating size and shape consistency of the AuNPs. Also, the only peak of the absorbance spectra indicated that particles obtained by the microreactor system exhibit a uniform globular shape and no other shaped gold nanoparticle such as triangles were observed (Amendola et al. 2017). SEM image showed that the synthesized AuNPs were quasi-spherical with a diameter of $22.09 \pm 2.26 \mathrm{~nm}$ (Figure S3). For comparison, syntheses with the same conditions were done using the previous $2 \mathrm{D}$ microreactor. As shown in Fig. 6b, the signal of the first synthesis was lower which means that part of the nanoparticles were fouling in the channel. The second synthesis showed a broad band indicating aggregated AuNPs from the last synthesis which were blocked in the channel. After that, the synthesis was still not repeatable as to be seen by a broad band. The 3D microreactor was then applied for synthesis of AuNPs to investigate the effects of different parameters.

\subsubsection{Effect of the $\mathrm{pH}$ of the reducing agent}

The size of the AuNPs mainly depends on the initial $\mathrm{pH}$ of the AA solution (Wagner et al. 2005). AA with higher $\mathrm{pH}$ possesses a higher reducing power than with lower $\mathrm{pH}$. In this case, increasing the $\mathrm{pH}$ of AA can result in a faster reaction rate and more nucleation sites, and consequently in smaller sizes and higher numbers. The UV-Vis absorbance spectra are shown in Fig. 7a. From the overall view, there was a shift to a lower wavelength from 563 to $517 \mathrm{~nm}$ with the increase of the initial $\mathrm{pH}$ of the AA solution indicating the decrease in size of the AuNPs. The size distribution can be studied by measuring the full-width at half-maximum

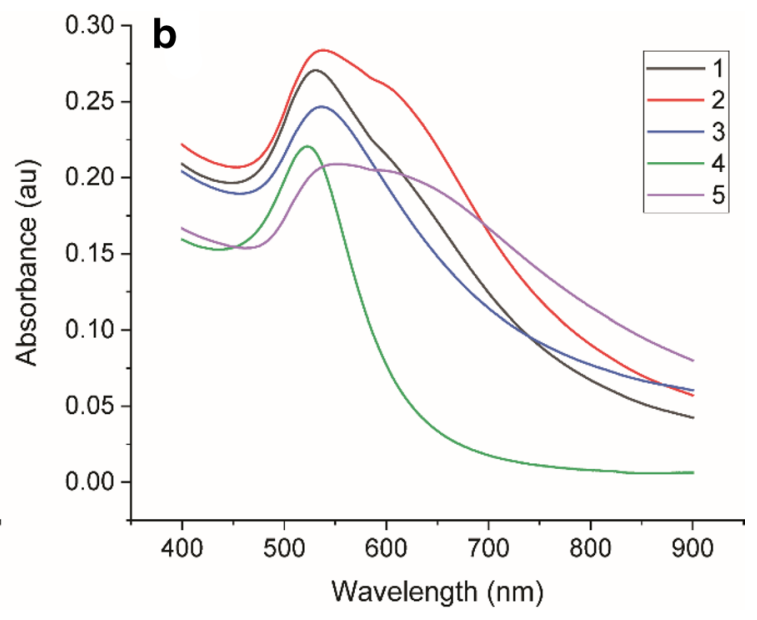

figures represent the number of synthesis. The reactant stream flow rates $\left(\mathrm{HAuCl}_{4} / \mathrm{AA}\right)$ were $3 / 30 \mu \mathrm{l} / \mathrm{s}$. The $\mathrm{pH}$ of the reducing agent was 8.46

(FWHM) of SPR band (Tran et al. 2016). The resulting spectra can be separated into three areas-low $\mathrm{pH}$ area $(\mathrm{pH} 4.30$, 5.66), middle $\mathrm{pH}$ area $(\mathrm{pH} 7.10,7.61)$ and high $\mathrm{pH}$ area $(\mathrm{pH} 10.32,10.91)$. The peaks in the middle $\mathrm{pH}$ area were narrowest with FWHM of about $50 \mathrm{~nm}$ which meant sizes of AuNPs were more uniform and focused. However, the spectra were wider in low and high $\mathrm{pH}$ areas with FWHM of around $90 \mathrm{~nm}$ even though they biased to the corresponding position (low $\mathrm{pH}$ to high wavelength and high $\mathrm{pH}$ to low wavelength). As shown in Figure S4, the AuNPs synthesized at low $\mathrm{pH}$ were large in size. And the size distribution was not uniform at both high and low $\mathrm{pH}$ values. Therefore, the medium $\mathrm{pH}$ was more suitable for the synthesis of uniform AuNPs. The divergent size distribution of AuNPs may be related to different ion species of AA in a variation of the $\mathrm{pH}$ solutions (Mukai et al. 1991). $\mathrm{AsH}_{2}$ dominates the solution at lower $\mathrm{pH}$ values up to 4 , while the percentage of $\mathrm{AsH}^{-}$increases to reach $100 \%$ when the $\mathrm{pH}$ is approximately 8. When the $\mathrm{pH}$ is $8, \mathrm{AsH}_{2}$ reaches its minimum concentration. With increase of $\mathrm{pH}$, the percentage of $\mathrm{AsH}^{-}$starts to decrease and percentage of $\mathrm{As}_{2}{ }^{-}$ions start to increase. Apparently, the mixture of different species of ascorbic acid affect the growth pattern.

\subsubsection{Effect of the reagent concentrations}

The concentration of reagents may affect the size of AuNPs. In Fig. 7b, the increased concentration of AA caused a shift to lower wavelength with higher reducing power. In Fig. 7c, with an increase of the concentration of gold precursor, the intensity of the absorbance maxima increased as well, which means that more AuNPs were synthesized. At the same time, the spectra were shifted from 540 to $529 \mathrm{~nm}$ and the FWHM 

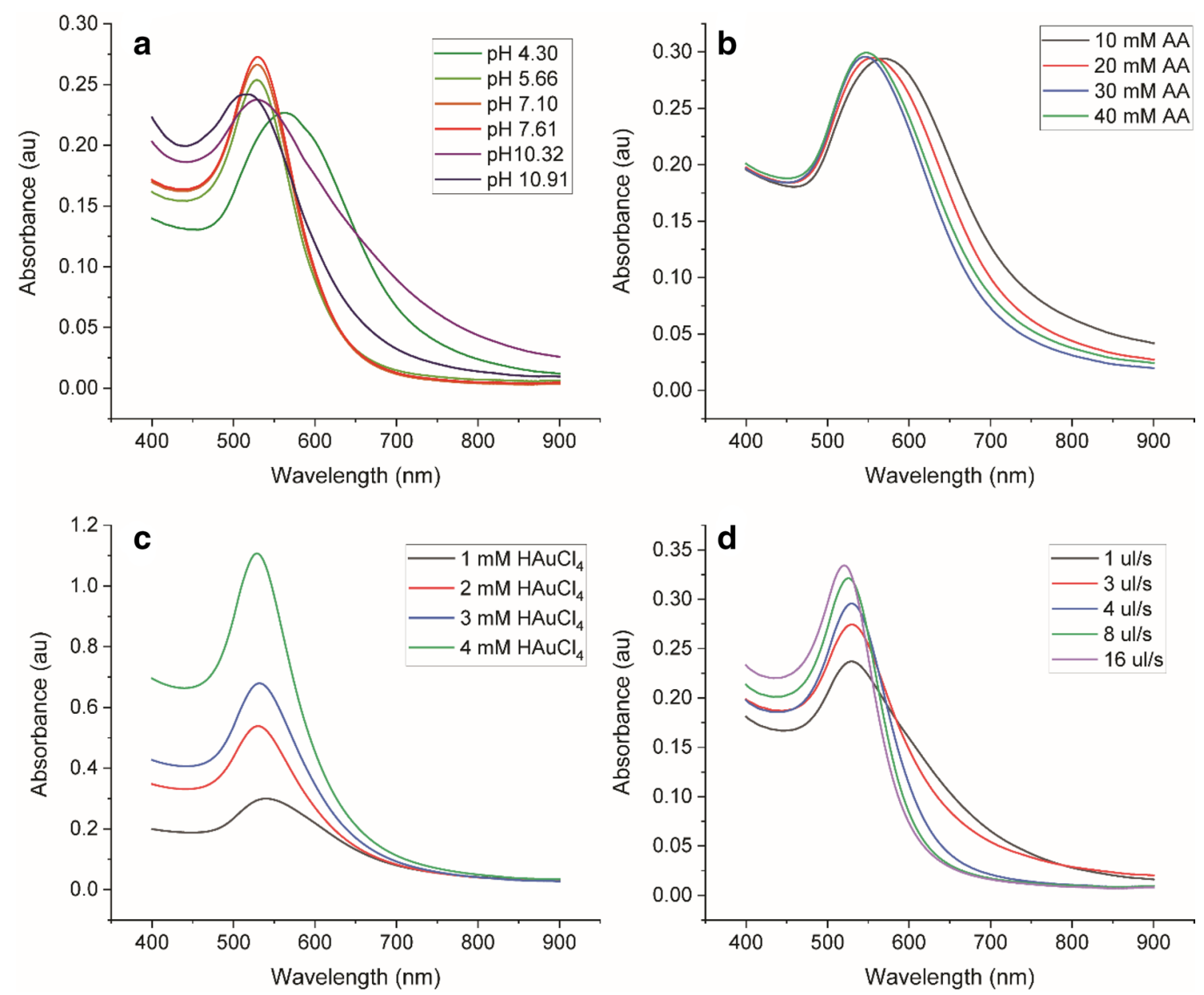

Fig. 7 UV-Vis spectra of gold nanoparticle suspension prepared by a keeping flow rate constant at $5 \mathrm{ul} / \mathrm{s}$ and changing $\mathrm{pH}$ of reducing agent, $\mathbf{b}$ different concentrations of AA, $\mathbf{c}$ different concentrations of

gold precursor, $\mathbf{d}$ keeping the flow rate ratio (gold precursor: reducing reagents, 1:10) and changing the flow rate of gold precursor

decreased from 97 to $64 \mathrm{~nm}$. This implies a smaller size and monodispersity of the nanoparticles. Due to the higher concentration of gold precursor, more nucleation sites are offered. This result also indicates that the amount of AA is sufficient for reducing.

\subsubsection{Effect of the flow rate on nanoparticle synthesis}

The UV-Vis absorbance spectra using different flow rates are shown in Fig. 7d. With increase of the flow rate, the spectra are shifted from 530 to $518 \mathrm{~nm}$ and the maximum absorbance increased by $40.8 \%$, which indicate a smaller size and higher concentration of nanoparticles. The increased flow rate of both regents was likely to increase the diffusion of AA into $\mathrm{HAuCl}_{4}$ solution which in turn could increase the nucleation rate (Jun et al. 2012). The more nucleation sites, the higher number of nanoparticles. With the same amount of gold precursor, a smaller size of nanoparticles

was generated. Therefore, smaller size and higher number concentration of AuNPs were achieved with higher flow rate. However, when the flow rate was high enough, the effect was not significant compared with other factors, as shown in Figure S5.

\section{Conclusions}

A cheap, easy and fast fabrication method for multilayer laminated 3D PMMA-PSA microfluidic chips was presented in this work. Sheet layers were cut by a cutting plotter which allows a fast fabrication of microfluidic structures for different applications. The assembly of the devices was carried out manually with a simple alignment tool. No thermocompression was required and only a soft compression by a roller was sufficient. The complete fabrication process from device design concept to working device can be completed in minutes without the need of expensive equipment. 
This fabrication protocol was used for the generation of a 3D microreactor for the reproducible synthesis of AuNPs. Compared with a 2D microreactor, the sheath stream can focus the central flow as well as avoid fouling. The proposed nonlithographic approach offers an alternative for institutes, where specialized microfabrication equipment is not available. The results presented in this paper show the successfully reproducible synthesis of spherical colloidal AuNPs without fouling using this 3D hydrodynamic flow-focused, laminated microfluidic device. This was an improvement in comparison to conventional batch reactors, where the properties of nanoparticles vary from batch-to-batch. Also, the microfluidic reactor makes it possible to do online synthesis and characterizing or detection (Pahl et al. 2019). This method of flow-based plastic microfluidic chips construction can be adapted for methods of many different fields, such as pharmaceutical analysis (Cui and Wang 2019), cellomics (Andersson and Berg 2003) and flow cytometry (Ateya et al. 2008; Shrirao et al. 2018).

Supplementary Information The online version contains supplementary material available at https://doi.org/10.1007/s10404-021-02463-6.

Acknowledgements Special thanks to Jonas Bemetz for helping Matlab programme and build automatic synthesis setup. We also thank Roland Hopper from workshop for the fabrication of the PMMA carrier. We appreciate Katharina Sollweck for linguistic assistance and helpful suggestions during the preparation of this manuscript. Many thanks to Christine Benning for SEM measurement.

Author contributions Conceptualization of the project was done by YW and MS. The manuscript was written by YW. Experiments were performed by YW under supervision of MS. MS reviewed the manuscript, acquired funding and supervised YW. All authors have given approval to the final version of the manuscript.

Funding Open Access funding enabled and organized by Projekt DEAL. This research was funded by the China Scholarship Council (CSC) with number 201706210083

\section{Declaration}

Conflicts of interest The authors declare that they have no conflicts to declare.

Open Access This article is licensed under a Creative Commons Attribution 4.0 International License, which permits use, sharing, adaptation, distribution and reproduction in any medium or format, as long as you give appropriate credit to the original author(s) and the source, provide a link to the Creative Commons licence, and indicate if changes were made. The images or other third party material in this article are included in the article's Creative Commons licence, unless indicated otherwise in a credit line to the material. If material is not included in the article's Creative Commons licence and your intended use is not permitted by statutory regulation or exceeds the permitted use, you will need to obtain permission directly from the copyright holder. To view a copy of this licence, visit http://creativecommons.org/licenses/by/4.0/.

\section{References}

Amendola V, Pilot R, Frasconi M, Marago OM, Iati MA (2017) Surface plasmon resonance in gold nanoparticles: a review. J Phys Condens Matter 29:203002. https://doi.org/10.1088/1361-648X/ aa60f3

Andersson H, van den Berg A (2003) Microfluidic devices for cellomics: a review. Sens Actuat 92:315-325. https://doi.org/10. 1016/s0925-4005(03)00266-1

Ateya DA, Erickson JS, Howell PB Jr, Hilliard LR, Golden JP, Ligler FS (2008) The good, the bad, and the tiny: a review of microflow cytometry. Anal Bioanal Chem 391:1485-1498. https://doi.org/ 10.1007/s00216-007-1827-5

Bandulasena MV, Vladisavljević GT, Odunmbaku OG, Benyahia B (2017) Continuous synthesis of PVP stabilized biocompatible gold nanoparticles with a controlled size using a 3D glass capillary microfluidic device. Chem Eng Sci 171:233-243. https://doi. org/10.1016/j.ces.2017.05.035

Bartholomeusz DA, Boutté RW, Andrade JD (2005) Xurography- rapid prototyping of microstructures. J Microelectromech Syst. https:// doi.org/10.1109/JMEMS.2005.859087

Bemetz J, Wegemann A, Saatchi K, Haase A, Hafeli UO, Niessner R, Gleich B, Seidel M (2018) Microfluidic-based synthesis of magnetic nanoparticles coupled with miniaturized NMR for online relaxation studies. Anal Chem 90:9975-9982. https://doi.org/10. 1021/acs.analchem.8b02374

Chen C, Mehl BT, Munshi AS, Townsend AD, Spence DM, Martin RS (2016a) 3D-printed microfluidic devices: fabrication, advantages and limitations-a mini review. Anal Methods 8:6005-6012. https://doi.org/10.1039/C6AY01671E

Chen X, Shen J, Zhou M (2016b) Rapid fabrication of a four-layer PMMA-based microfluidic chip using $\mathrm{CO}_{2}$-laser micromachining and thermal bonding. J Micromech Microeng 26:107001. https:// doi.org/10.1088/0960-1317/26/10/107001

Chen X, Li T, Gao QI (2019) A novel method for rapid fabrication of Pmma microfluidic chip by laser cutting and sealing integration. Surf Rev Lett. https://doi.org/10.1142/s0218625x19500422

Cosson S, Aeberli LG, Brandenberg N, Lutolf MP (2015) Ultra-rapid prototyping of flexible, multi-layered microfluidic devices via razor writing. Lab Chip 15:72-76. https://doi.org/10.1039/c4lc0 0848k

Cui P, Wang S (2019) Application of microfluidic chip technology in pharmaceutical analysis: a review. J Pharm Anal 9:238-247. https://doi.org/10.1016/j.jpha.2018.12.001

Faustino V, Catarino SO, Lima R, Minas G (2016) Biomedical microfluidic devices by using low-cost fabrication techniques: a review. J Biomech 49:2280-2292. https://doi.org/10.1016/j.jbiomech. 2015.11.031

Gomez-de Pedro S, Puyol M, Alonso-Chamarro J (2010) Continuous flow synthesis of nanoparticles using ceramic microfluidic devices. Nanotechnology 21:415603. https://doi.org/10.1088/ 0957-4484/21/41/415603

Hernández-Rodríguez JF, Rojas D, Escarpa A (2020) Rapid and costeffective benchtop microfabrication of disposable carbon-based electrochemical microfluidic devices. Sens Actuat. https://doi.org/ 10.1016/j.snb.2020.128679

Hizawa T, Takano A, Parthiban P, Doyle PS, Iwase E, Hashimoto M (2018) Rapid prototyping of fluoropolymer microchannels by xurography for improved solvent resistance. Biomicrofluidics 12:064105. https://doi.org/10.1063/1.5051666

Jafek AR, Harbertson S, Brady H, Samuel R, Gale BK (2018) Instrumentation for xPCR Incorporating qPCR and HRMA. Anal Chem 90:7190-7196. https://doi.org/10.1021/acs.analchem.7b05176

Jun H, Fabienne T, Florent M, Coulon PE, Nicolas M, Olivier S (2012) Understanding of the size control of biocompatible gold 
nanoparticles in millifluidic channels. Langmuir 28:15966-15974. https://doi.org/10.1021/la303439f

Kim P (2008) Soft lithography for microfluidics: a review. BioChip J $2: 1-11$

Levis M, Kumar N, Apakian E, Moreno C, Hernandez U, Olivares A, Ontiveros F, Zartman JJ (2019) Microfluidics on the fly: inexpensive rapid fabrication of thermally laminated microfluidic devices for live imaging and multimodal perturbations of multicellular systems. Biomicrofluidics 13:024111. https://doi.org/10.1063/1. 5086671

Luty-Błocho M, Wojnicki M, Fitzner K (2017) Gold nanoparticles formation via $\mathrm{Au}(\mathrm{III})$ complex ions reduction with 1-ascorbic acid. Int J Chem Kinet 49:789-797. https://doi.org/10.1002/kin.21115

Ma Y, Cao X, Feng X, Ma Y, Zou H (2007) Fabrication of superhydrophobic film from PMMA with intrinsic water contact angle below $90^{\circ}$. Polymer 48:7455-7460. https://doi.org/10.1016/j. polymer.2007.10.038

Migneault S, Koubaa A, Erchiqui F, Chaala A, Englund K, Wolcott MP (2009) Effects of processing method and fiber size on the structure and properties of wood-plastic composites. Compos A Appl Sci Manuf 40:80-85. https://doi.org/10.1016/j.compositesa. 2008.10.004

Mukai K, Nishimura M, Kikuchi S (1991) Stopped-flow investigation of the reaction of vitamin $\mathrm{C}$ with tocopheroxyl radical in aqueous triton X-100 micellar solutions. The structure-activity relationship of the regeneration reaction of tocopherol by vitamin C. J Biol Chem 266:274-278

Nath P, Fung D, Kunde YA, Zeytun A, Branch B, Goddard G (2010) Rapid prototyping of robust and versatile microfluidic components using adhesive transfer tapes. Lab Chip 10:2286-2291. https://doi. org/10.1039/c002457k

Pahl M, Mayer M, Schneider M, Belder D, Asmis KR (2019) Joining microfluidics with infrared photodissociation: online monitoring of isomeric flow-reaction intermediates. Anal Chem 91:31993203. https://doi.org/10.1021/acs.analchem.8b05532

Patel JN, Kaminska B, Gray BL, Gates BD (2008) PDMS as a sacrificial substrate for SU-8-based biomedical and microfluidic applications. J Micromech Microeng 18:095028. https://doi.org/10.1088/ 0960-1317/18/9/095028

Rhee M, Valencia PM, Rodriguez MI, Langer R, Farokhzad OC, Karnik R (2011) Synthesis of size-tunable polymeric nanoparticles enabled by 3D hydrodynamic flow focusing in single-layer microchannels. Adv Mater 23:H79-83. https://doi.org/10.1002/ adma.201004333
Rothstein JP (2010) Slip on superhydrophobic surfaces. Annu Rev Fluid Mech 42:89-109. https://doi.org/10.1146/annur ev-fluid-121108-145558

Schoenitz M, Grundemann L, Augustin W, Scholl S (2015) Fouling in microstructured devices: a review. Chem Commun (camb) 51:8213-8228. https://doi.org/10.1039/c4cc07849g

Shalom D, Wootton RCR, Winkle RF, Cottam BF, Vilar R, deMello AJ, Wilde CP (2007) Synthesis of thiol functionalized gold nanoparticles using a continuous flow microfluidic reactor. Mater Lett 61:1146-1150. https://doi.org/10.1016/j.matlet.2006.06.072

Shi L, Buhler E, Boue F, Carn F (2017) How does the size of gold nanoparticles depend on citrate to gold ratio in Turkevich synthesis? Final answer to a debated question. J Colloid Interface Sci 492:191-198. https://doi.org/10.1016/j.jcis.2016.10.065

Shrirao AB, Fritz Z, Novik EM, Yarmush GM, Schloss RS, Zahn JD, Yarmush ML (2018) Microfluidic flow cytometry: the role of microfabrication methodologies, performance and functional specification. Technology (singap World Sci) 6:1-23. https://doi. org/10.1142/S2339547818300019

Song S, Lee KY (2006) Polymers for microfluidic chips. Macromol Res 14:121-128. https://doi.org/10.1007/bf03218498

Tran M, DePenning R, Turner M, Padalkar S (2016) Effect of citrate ratio and temperature on gold nanoparticle size and morphology. Mater Res Express. https://doi.org/10.1088/2053-1591/3/ $10 / 105027$

Vullev VI, Wan J, Heinrich V, Landsman P, Bower PE, Xia B, Millare $B$, Jones G 2nd (2006) Nonlithographic fabrication of microfluidic devices. J Am Chem Soc 128:16062-16072. https://doi.org/10. 1021/ja061776o

Wagner J, Kohler JM (2005) Continuous synthesis of gold nanoparticles in a microreactor. Nano Lett 5:685-691. https://doi.org/10. $1021 / \mathrm{n} 1050097 \mathrm{t}$

Wagner J, Kirner T, Mayer G, Albert J, Köhler JM (2004) Generation of metal nanoparticles in a microchannel reactor. Chem Eng J 101:251-260. https://doi.org/10.1016/j.cej.2003.11.021

Publisher's Note Springer Nature remains neutral with regard to jurisdictional claims in published maps and institutional affiliations. 